\title{
Energy-Momentum Distribution of the Weyl-Lewis-Papapetrou and the Levi-Civita Metrics
}

\author{
M. Sharif \\ Department of Mathematics, University of the Punjab, \\ Quaid-e-Azam Campus Lahore-54590, PAKISTAN
}

Received on 3 October, 2007

\begin{abstract}
This paper is devoted to compute the energy-momentum densities for two exact solutions of the Einstein field equations by using the prescriptions of Einstein, Landau-Lifshitz, Papapetrou and Möller. The spacetimes under consideration are the Weyl-Lewis-Papapetrou and the Levi-Civita metrics. The Weyl metric becomes the special case of the Weyl-Lewis-Papapetrou solution. The Levi-Civita metric provides constant momentum in each prescription with different energy density. The Weyl-Lewis-Papapetrou metric yields all the quantities different in each prescription. These differences support the well-defined proposal developed by Cooperstock and from the energy-momentum tensor itself.
\end{abstract}

Keywords: Energy-Momentum Distribution

\section{INTRODUCTION}

The relativistic analogues of the classical principle of the conservation of energy and momentum can be obtained with the help of the well-known equation [1]

$$
\frac{\partial}{\partial x^{b}}\left(\mathfrak{I}_{a}^{b}+t_{a}^{b}\right)=0, \quad(a, b=0,1,2,3),
$$

where $\mathfrak{I}_{a}^{b}$ is a tensor density of material energy and momentum and $t_{a}^{b}$ is the pseudo-tensor density of gravitational energy and momentum. The definition of localized energy density is a longstanding problem [2] in General Relativity (GR). On the basis of the principle of equivalence, it is usually assumed that the gravitational energy cannot be localized. The principle of equivalence is frequently invoked to ensure that the gravitational field can be made vanish in a sufficiently small region of the spacetime. Misner at el. [3] showed that the energy can only be localized in spherical systems. But later on, Cooperstock and Sarracino [4] proved that if energy is localizable for spherical systems, then it can be localized in any system. Much attention has been devoted for this problematic issue.

An energy-momentum complex is the sum of the energymomentum of matter and an appropriate pseudo-tensor. Einstein showed that the energy-momentum pseudo-complex provides satisfactory expression for the total energy and momentum of a closed system in the form of three dimensional integral. There are some drawbacks of the Einstein energymomentum complex. One of these drawbacks is that it is not symmetric in its indices. However, Landau-Lifshitz energymomentum complex satisfies this requirement. In order to determine the conserved total four-momentum for gravitation with matter, Landau-Lifshitz introduced a system of coordinates at some particular point in spacetime for which all the first derivatives of the metric tensor vanish. Papapetrou energy-momentum complex is the least known among the four definitions under discussion and as a result, it has been re-discovered several times. Although the Einstein energymomentum complex provides useful expression for the total energy-momentum of a closed system. However, from the GR viewpoint, Möller argued that it is unsatisfactory to transform a system into quasi-Cartesian coordinates. Möller tried to find out an expression of energy-momentum which is independent of the choice of particular coordinate system.

Einstein was the first to construct a locally conserved energy-momentum complex [5]. After this attempt, many physicists including Tolman [6], Landau-Lifshitz [7], Papapetrou [8], Bergmann [9] and Weinberg [10] introduced different definitions for the energy-momentum complex. These definitions can only give meaningful results if the calculations are performed in Cartesian coordinates. In 1990, Bondi [11] argued that a non-localizable form of energy is not allowed in GR. After this, the idea of quasi-local energy was introduced by Penrose and other researchers [12-14]. In this method, one can use any coordinate system while finding the quasi-local masses to obtain the energy-momentum of a curved spacetime. Bergqvist [15] considered seven different definitions of quasi-local mass and showed that no two of these definitions give the same result. Chang at el. [16] showed that every energy-momentum complex can be associated with a particular Hamiltonian boundary term and hence the energymomentum complexes may also be considered as quasi-local.

Möller [17,18] proposed an expression which is the best to make calculations in any coordinate system. He claimed that his expression would give the same results for the total energy and momentum as the Einstein's energy-momentum complex for a closed system. Lessner [19] gave his opinion that Möller's definition is a powerful concept of energy and momentum in GR. However, Möller's prescription was also criticized by some people [11,20,21]. Komar's complex [21], though not restricted to the use of Cartesian coordinates, is not applicable to non-static spacetimes. Thus each of these energy-momentum complex has its own drawbacks. As a result, these ideas of the energy-momentum complexes could not lead to some unique definition of energy in GR.

Virbhadra [22] generated interest on this topic by using different prescriptions to calculate energy-momentum of a spacetime. He found that different prescriptions could lead to the same result if appropriate coordinates are used. Aguirregabiria et al. [23] showed that five different energy-momentum complexes gave the same result for any Kerr-Schild class (including the Schwarzchild, ReissnerNordström, Kerr and Vaidya metrics). Xulu [24,25] extended 
this investigation and found same energy distribution in the Melvin magnetic and Bianchi type I universe. Chamorro and Virbhadra [26] and Xulu [27] studied the energy distribution of charged black holes with a dilaton field.

It was hoped [25] that some particular properties might give a basis to believe that some pseudo-tensors of energymomentum density had a special meaning. However, there exists examples of spacetimes which do not support this viewpoint. In this regard, Sharif [28,29] considered the class of gravitational waves and Gödel universe and used the four definitions of energy-momentum. He concluded that results obtained are not consistent in different prescriptions. Recently, Sharif and Fatima [30,31] considered some more examples of Non-Null Einstein-Maxwell solution, singularity-free cosmological model and Weyl metrics and applied four different complexes. They found that the energy-momentum complexes do not provide the same results for any of these spacetimes. This paper continues the investigation by considering two more examples.

The paper is organized as follows. In section 2, we shall briefly mention different prescriptions to evaluate energymomentum distribution. Sections 3 and 4 are devoted for the evaluation of energy-momentum densities for the two particular spacetimes using the prescriptions of Einstein, LandauLifshitz, Papapetrou and Möller. Finally, in the last section, we shall discuss and summarize all the results obtained.

\section{ENERGY-MOMENTUM COMPLEXES}

In this section, we shall elaborate four different approaches to evaluate the energy-momentum density components of different spacetimes.

\section{A. Einstein Energy-Momentum Complex}

The energy-momentum complex of Einstein [2] is given by

$$
\Theta_{a}^{b}=\frac{1}{16 \pi} H_{a, c}^{b c}, \quad(a, b, \ldots=0,1,2,3),
$$

where

$$
H_{a}^{b c}=\frac{g_{a d}}{\sqrt{-g}}\left[-g\left(g^{b d} g^{c e}-g^{c d} g^{b e}\right)\right]_{, e}
$$

It is to be noted that $H_{a}^{b c}$ is anti-symmetric in indices $b$ and $c$. $\Theta_{0}^{0}$ is the energy density, $\Theta_{0}^{i}(i=1,2,3)$ are the components of momentum density and $\Theta_{i}^{0}$ are the energy current density components. Einstein showed that the energy-momentum pseudo-complex $\Theta_{a}^{b}$ provides satisfactory expression for the total energy and momentum of closed system in the form of 3-dimensional integral.

\section{B. Landau-Lifshitz Energy-Momentum Complex}

There were some drawbacks of Einstein energy-momentum complex. One main drawback was that it was not symmetric in its indices. As a result, this cannot be used to define conservation laws of angular momentum. However, Landau-Lifshitz energy-momentum complex is symmetric and they are able to develop a conserved angular momentum complex in addition to that of energy-momentum. The energy-momentum complex of Landau-Lifshitz [7] is given by

$$
L^{a b}=\frac{1}{16 \pi} \ell_{, c d}^{a c b d}
$$

where

$$
\ell^{a c b d}=-g\left(g^{a b} g^{c d}-g^{a d} g^{c b}\right) .
$$

$L^{00}$ represents the energy density of the whole system including gravitation and $L^{o i}$ represent the components of the momentum density. $\ell^{a b c d}$ has symmetries of the Riemann curvature tensor. It is clear from Eq.(3) that $L^{a b}$ is symmetric with respect to its indices.

\section{Papapetrou Energy-Momentum Complex}

Papapetrou energy-momentum complex is the least known among the four definitions under discussion, as a result, it has been re-discovered several times. The expression was found using the generalized Belinfante method. The symmetric energy-momentum complex of Papapetrou [8] is given as

$$
\Omega^{a b}=\frac{1}{16 \pi} N_{, c d}^{a b c d},
$$

where

$$
N^{a b c d}=\sqrt{-g}\left(g^{a b} \eta^{c d}-g^{a c} \eta^{b d}+g^{c d} \eta^{a b}-g^{b d} \eta^{a c}\right),
$$

and $\eta^{a b}$ is the Minkowski spacetime. The quantities $N^{a b c d}$ are symmetric in its first two indices $a$ and $b$. The locally conserved quantities $\Omega^{a b}$ contain contribution from the matter, non-gravitational and gravitational field. The quantity $\Omega^{00}$ represents energy density and $\Omega^{0 i}$ are the momentum density components.

\section{Möller Energy-Momentum Complex}

Although the Einstein energy-momentum complex provides useful expression for the total energy-momentum of a closed system. However, from the GR viewpoint, Möller [17] argued that it is unsatisfactory to transform a system into quasi-Cartesian coordinates. Möller tried to find out an expression of energy-momentum which is independent of the choice of particular coordinate system. His energymomentum complex is given by

$$
M_{a}^{b}=\frac{1}{8 \pi} K_{a, c}^{b c},
$$

where

$$
K_{a}^{b c}=\sqrt{-g}\left(g_{a d, e}-g_{a e, d}\right) g^{b e} g^{c d} .
$$


Here $K_{a}^{b c}$ is antisymmetric, $M_{0}^{0}$ is the energy density, $M_{0}^{i}$ are the momentum density components and $M_{i}^{0}$ are the energy current density components. In the next two sections, we apply these prescriptions to evaluate energy-momentum distribution for two particular examples.

\section{WEYL-LEWIS-PAPAPETROU METRIC}

The class of stationary axisymmetric solutions of the Einstein field equations is the appropriate framework for the attempts to include the gravitational effect of an external source in an exact analytical manner [32]. At the same time, such spacetimes are of obvious astrophysical importance, as they describe the exterior of the body in equilibrium. The complete family of exact solutions representing accelerating and rotating black holes with possible electromagnetic charges and a nut parameter is known in terms of a modified Plebanskidemianski metric. This demonstrates the singularity and horizon structure of the sources but not that the complete spacetime describes two causally separated black holes. To demonstrate this property, the metric was first cast in the WeylLewis-Papapetrou form. The line element of stationary axisymmetric spacetime of the Weyl-Lewis-Papapetrou metric is given by [33]

$$
d s^{2}=e^{2 \psi}(d t-\omega d \phi)^{2}-e^{2(\gamma-\psi)}\left(d \rho^{2}+d z^{2}\right)-\rho^{2} e^{-2 \psi} d \phi^{2}
$$

where $\omega$ is the angular velocity and $\gamma, \psi, \omega$ are functions of $\rho$ and $\mathrm{z}$ only. It is mentioned here this reduces to the Weyl metric for $\omega=0$. To get meaningful results in Einstein, LandauLifshitz and Papapetrou prescriptions, we transform this metric into Cartesian coordinates given by

$$
\begin{aligned}
d s^{2} & =e^{2 \psi} d t^{2}+\left(\omega^{2} e^{2 \psi}-\rho^{2} e^{-2 \psi}\right)\left(\frac{x d y-y d x}{\rho^{2}}\right)^{2}-e^{2(\gamma-\psi)}\left(\frac{x d x+y d y}{\rho}\right)^{2} \\
& -2 \omega e^{2 \psi}\left(\frac{x d y-y d x}{\rho^{2}}\right) d t-e^{2(\gamma-\psi)} d z^{2}
\end{aligned}
$$

\section{A. Energy-Momentum Densities in Einstein Complex}

The energy-momentum densities of the Weyl-Lewis-Papapetrou metric can be found by Einstein complex with the components of $H_{a}^{b c}$ that can be computed by using Eq.(2). When we make use of these components in Eq.(1), we obtain the following components of energy, momentum and energy current densities

$$
\begin{aligned}
& \Theta_{0}^{0}=\frac{1}{8 \pi \rho}\left[\gamma_{\rho}\left(e^{2 \gamma}-1\right)-\rho \gamma_{\rho \rho}+2 \psi_{\rho}+2 \rho \psi_{\rho \rho}-\rho \gamma_{z z}+2 \rho \psi_{z z}\right. \\
& \left.+\frac{\omega_{\rho}^{2} e^{4 \psi}}{2 \rho}+\frac{\omega \omega_{\rho} e^{4 \psi}}{2 \rho}-\frac{\omega \omega_{\rho} e^{4 \psi}}{2 \rho^{2}}+\frac{2 \omega \omega_{\rho} \psi_{\rho} e^{4 \psi}}{\rho}\right] \\
& \Theta_{1}^{0}=\frac{y}{16 \pi \rho^{2}}\left[\left(\omega_{\rho \rho}+\omega_{z z}\right)+2 \omega\left(\gamma_{\rho \rho}+\gamma_{z z}\right)+2\left(\omega_{\rho} \gamma_{\rho}+\omega_{z} \gamma_{z}\right)\right. \\
& -2 \omega\left\{2\left(\gamma_{\rho \rho}-2 \psi_{\rho \rho}\right)+\left(\gamma_{z z}-2 \psi_{z z}\right)\right\}-2 \omega\left\{\left(\gamma_{\rho}-2 \psi_{\rho}\right) \psi_{\rho}\right. \\
& \left.+\left(\gamma_{z}-2 \psi_{z}\right) \psi_{z}\right\}+\frac{4 \omega^{2} e^{4 \psi}}{\rho^{2}}\left(\omega_{\rho} \psi_{\rho}+\omega_{z} \psi_{z}\right) \\
& +\frac{\omega^{2} e^{4 \psi}}{\rho^{2}}\left(\omega_{\rho \rho}+\omega_{z z}\right)+\frac{2 \omega e^{4 \psi}}{\rho}\left(\omega_{\rho}^{2}+\omega_{z}^{2}\right)-\frac{2 \omega_{\rho}}{\rho}+\frac{2 \omega}{\rho^{2}} \\
& \left.+\frac{\omega_{\rho} e^{2 \gamma}}{\rho}+\frac{2 \omega \gamma_{\rho} e^{2 \gamma}}{\rho}-\frac{2 \omega e^{2 \gamma}}{\rho^{2}}-\frac{2 \omega^{2} \omega_{\rho} e^{4 \psi}}{\rho^{3}}\right], \\
& \Theta_{2}^{0}=-\frac{x}{16 \pi \rho^{2}}\left[\left(\omega_{\rho \rho}+\omega_{z z}\right)+2 \omega\left(\gamma_{\rho \rho}+\gamma_{z z}\right)+2\left(\omega_{\rho} \gamma_{\rho}+\omega_{z} \gamma_{z}\right)\right. \\
& -2 \omega\left\{2\left(\gamma_{\rho \rho}-2 \psi_{\rho \rho}\right)+\left(\gamma_{z z}-2 \psi_{z z}\right)\right\}-2 \omega\left\{\left(\gamma_{\rho}-2 \psi_{\rho}\right) \psi_{\rho}\right. \\
& \left.+\left(\gamma_{z}-2 \psi_{z}\right) \psi_{z}\right\}+\frac{4 \omega^{2} e^{4 \psi}}{\rho^{2}}\left(\omega_{\rho} \psi_{\rho}+\omega_{z} \psi_{z}\right) \\
& +\frac{\omega^{2} e^{4 \psi}}{\rho^{2}}\left(\omega_{\rho \rho}+\omega_{z z}\right)+\frac{2 \omega e^{4 \psi}}{\rho}\left(\omega_{\rho}^{2}+\omega_{z}^{2}\right)-\frac{2 \omega_{\rho}}{\rho}+\frac{2 \omega}{\rho^{2}} \\
& \left.+\frac{\omega_{\rho} e^{2 \gamma}}{\rho}+\frac{2 \omega \gamma_{\rho} e^{2 \gamma}}{\rho}-\frac{2 \omega e^{2 \gamma}}{\rho^{2}}-\frac{2 \omega^{2} \omega_{\rho} e^{4 \psi}}{\rho^{3}}\right] \text {, }
\end{aligned}
$$




$$
\begin{gathered}
\Theta_{0}^{1}=-\frac{y e^{4 \psi}}{16 \pi \rho^{2}}\left[\left(\omega_{\rho \rho}+\omega_{z z}\right)+4\left(\omega_{\rho} \psi_{\rho}+\omega_{z} \psi_{z}\right)\right] \\
\Theta_{0}^{2}=\frac{x e^{4 \psi}}{16 \pi \rho^{2}}\left[\left(\omega_{\rho \rho}+\omega_{z z}\right)+4\left(\omega_{\rho} \psi_{\rho}+\omega_{z} \psi_{z}\right)\right] \\
\Theta_{3}^{0}=0=\Theta_{0}^{3} .
\end{gathered}
$$

\section{B. Energy-Momentum Densities in Landau-Lifshitz Complex}

The non-zero components of $\ell^{a b c d}$ can be found by using Eq.(4) and consequently the components of energy and momentum (energy current) densities in Landau-Lifshitz prescription turn out to be

$$
\begin{aligned}
L^{00} & =\frac{1}{16 \pi \rho^{2}}\left[4 \rho\left(\gamma_{\rho}-\psi_{\rho}\right) e^{(\gamma-\psi)}-4 \rho\left(\gamma_{\rho}-2 \psi_{\rho}\right) e^{2(\gamma-2 \psi)}\right. \\
- & 2\left\{\left(\gamma_{\rho \rho}-2 \psi_{\rho \rho}\right)+\left(\gamma_{z z}-2 \psi_{z z}\right)\right\} e^{2(\gamma-2 \psi)}-4\left\{\left(\gamma_{\rho}-2 \psi_{\rho}\right)^{2}\right. \\
+ & \left.\left(\gamma_{z}-2 \psi_{z}\right)^{2}\right\} e^{(\gamma-2 \psi)}+\frac{2 e^{2 \gamma}}{\rho^{2}}\left\{\omega\left(\omega_{\rho \rho}+\omega_{z z}\right)+2\left(\omega_{\rho}^{2}+\omega_{z}^{2}\right)\right. \\
+ & 4 \omega\left(\omega_{\rho} \gamma_{\rho}+\omega_{z} \gamma_{z}\right)+\omega^{2}\left(\gamma_{\rho \rho}+\gamma_{z z}\right)+2 \omega^{2}\left(\gamma_{\rho}^{2}+\gamma_{z}^{2}\right) \\
- & \left.\left.\frac{2 \omega \omega_{\rho}}{\rho}-\frac{2 \omega^{2} \gamma_{\rho}}{\rho}+\frac{\omega^{2}}{\rho^{2}}\right\}\right], \\
L^{10} & =L^{01}=-\frac{y e^{2 \gamma}}{16 \pi \rho^{2}}\left[\left(\omega_{\rho \rho}+\omega_{z z}\right)+4\left(\omega_{\rho} \gamma_{\rho}+\omega_{z} \gamma_{z}\right)\right. \\
& \left.+2 \omega\left(\gamma_{\rho \rho}+\gamma_{z z}\right)+4 \omega\left(\gamma_{\rho}^{2}+\gamma_{z}^{2}\right)-\frac{\omega_{\rho}}{\rho}-\frac{2 \omega \gamma_{\rho}}{\rho}\right] \\
& \left.=2 \omega\left(\gamma_{\rho \rho}+\gamma_{z z}\right)+4 \omega\left(\gamma_{\rho}^{2}+\gamma_{z}^{2}\right)-\frac{\omega_{\rho}}{\rho}-\frac{2 \omega \gamma_{\rho}}{\rho}\right], \\
L^{20} & L^{02}=\frac{x e^{2 \gamma}}{16 \pi \rho^{2}}\left[\left(\omega_{\rho \rho}+\omega_{z z}\right)+4\left(\omega_{\rho} \gamma_{\rho}+\omega_{z} \gamma_{z}\right)\right. \\
& L^{03}=0 .
\end{aligned}
$$

\section{Energy-Momentum Densities in Papapetrou Complex}

Here the non-zero components of $N^{a b c d}$ are obtained with the help of Eq.(6). When we make use of these values in Eq.(5), it yields the following components of energy and momentum (energy current) densities

$$
\begin{aligned}
\Omega^{00} & =\frac{e^{2 \gamma}}{8 \pi \rho}\left[\left(1-e^{-4 \psi}\right) \gamma_{\rho}+\left\{2 \psi_{\rho}-\rho\left(\gamma_{\rho \rho}-2 \psi_{\rho \rho}+\gamma_{z z}-2 \psi_{z z}\right)\right\} e^{-4 \psi}\right. \\
& -2 \rho\left\{\left(\gamma_{\rho}-2 \psi_{\rho}\right)^{2}+\left(\gamma_{z}-2 \psi_{z}\right)^{2}\right\} e^{-4 \psi}+\frac{1}{\rho}\left(\omega_{\rho}^{2}+\omega_{z}^{2}\right) \\
& +\frac{2 \omega^{2}}{\rho}\left(\gamma_{\rho}^{2}+\gamma_{z}^{2}\right)+\frac{\omega}{\rho}\left(\omega_{\rho \rho}+\omega_{z z}\right)+\frac{\omega^{2}}{\rho}\left(\gamma_{\rho \rho}+\gamma_{z z}\right) \\
& \left.+\frac{4 \omega \omega_{\rho}}{\rho}\left(\omega_{\rho} \gamma_{\rho}+\omega_{z} \gamma_{z}\right)+\frac{2 \omega^{2}}{\rho^{3}}-\frac{3 \omega^{2} \gamma_{\rho}}{\rho^{2}}-\frac{3 \omega \omega_{\rho}}{\rho^{2}}\right]
\end{aligned}
$$




$$
\begin{gathered}
\Omega^{10}=\Omega^{01}=-\frac{y e^{2 \gamma}}{16 \pi \rho^{2}}\left[\left(\omega_{\rho \rho}+\omega_{z z}\right)+4\left(\omega_{\rho} \gamma_{\rho}+\omega_{z} \gamma_{z}\right)\right. \\
\left.+2 \omega\left(\gamma_{\rho \rho}+\gamma_{z z}\right)+4 \omega\left(\gamma_{\rho}^{2}+\gamma_{z}^{2}\right)-\frac{\omega_{\rho}}{\rho}-\frac{2 \omega \gamma_{\rho}}{\rho}\right], \\
\Omega^{20}=\Omega^{02}=\frac{x e^{2 \gamma}}{16 \pi \rho^{2}}\left[\left(\omega_{\rho \rho}+\omega_{z z}\right)+4\left(\omega_{\rho} \gamma_{\rho}+\omega_{z} \gamma_{z}\right)\right. \\
\left.+2 \omega\left(\gamma_{\rho \rho}+\gamma_{z z}\right)+4 \omega\left(\gamma_{\rho}^{2}+\gamma_{z}^{2}\right)-\frac{\omega_{\rho}}{\rho}-\frac{2 \omega \gamma_{\rho}}{\rho}\right], \\
\Omega^{30}=\Omega^{03}=0 .
\end{gathered}
$$

\section{Energy-Momentum Densities in Möller Complex}

This prescription does not require the transformation into Cartesian coordinates. The non-zero components of $K_{a}^{b c}$ are found from Eq.(8). Consequently, the components of energy, momentum and energy current densities become

$$
\begin{aligned}
& M_{0}^{0}= \frac{1}{4 \pi}\left(\psi_{\rho}+\rho \psi_{\rho \rho}+\rho \psi_{z z}\right)+\frac{e^{4 \psi}}{8 \pi \rho}\left[\omega\left(\omega_{\rho \rho}+\omega_{z z}\right)\right. \\
&+\left.4\left(\omega_{\rho} \psi_{\rho}+\omega_{z} \psi_{z}\right)+\left(\omega_{\rho}^{2}+\omega_{z}^{2}\right)-\frac{\omega \omega_{\rho}}{\rho}\right] \\
& M_{2}^{0}=-\frac{e^{4 \psi}}{8 \pi \rho}\left[\left(\omega^{2}+\rho^{2}\right)\left(\omega_{\rho \rho}+\omega_{z z}\right)\right. \\
&+ 4\left(\omega^{2}+\rho^{2}\right)\left(\omega_{\rho} \psi_{\rho}+\omega_{z} \psi_{z}\right)+2 \omega\left(\omega_{\rho}^{2}+\omega_{z}^{2}\right) \\
&+\left.4 \omega \rho^{2}\left(\psi_{\rho \rho}+\psi_{z z}\right)+4 \rho \omega \omega_{\rho}+\omega_{\rho}-\frac{\omega^{2} \omega_{\rho}}{\rho}\right] \\
& M_{0}^{2}= \frac{e^{4 \psi}}{8 \pi \rho}\left[\omega_{\rho \rho}+\omega_{z z}+4\left(\omega_{\rho} \psi_{\rho}+\omega_{z} \psi_{z}\right)-\frac{\omega_{\rho}}{\rho}\right] \\
& M_{1}^{0}=0=M_{0}^{1}=M_{3}^{0}=M_{0}^{3} .
\end{aligned}
$$

\section{THE LEVI-CIVITA METRIC}

The Levi-Civita metric is given by [34]

$$
d s^{2}=\rho^{4 s} d t^{2}-\rho^{4 s(2 s-1)}\left(d \rho^{2}+d z^{2}\right)-\alpha^{2} \rho^{2(1-2 s)} d \phi^{2},
$$

where $\alpha$ is a parameter and $s$ is a charge density parameter. The following interpretations are somewhat accepted for: $s=0, \frac{1}{2}$, this becomes locally flat spacetime, $s=0, \alpha=1$, this reduces to Minkowski spacetime and $s=0, \alpha \neq 1$, we have cosmic string.

One of the most interesting metrics of the family of the Weyl solutions is called $\gamma$-metric, also known as Zipoy-Voorhes metric [35]. The Levi-Civita metric can be obtained from a family of the Weyl-metric, i.e., the $\gamma$-metric by taking the limit when the length of its Newtonian image source tends to infinity. The line element can be transformed into Cartesian coordinates and is given by

$$
\begin{aligned}
d s^{2} & =\rho^{4 s} d t^{2}-\rho^{4 s(2 s-1)}\left(\frac{x d x+y d y}{\rho}\right)^{2} \\
& -\alpha^{2} \rho^{2(1-2 s)}\left(\frac{x d y-y d x}{\rho^{2}}\right)^{2}-\rho^{4 s(2 s-1)} d z^{2} .
\end{aligned}
$$

\section{A. Energy-Momentum Densities in Einstein Complex}

Using the components of $H_{a}^{b c}$, we obtain the components of energy-momentum

$$
\begin{aligned}
& \Theta_{0}^{0}=\frac{s^{2} \rho^{8 s^{2}-2}}{2 \pi \alpha}, \\
& \Theta_{i}^{0}=0=\Theta_{0}^{i}
\end{aligned}
$$

which gives constant momentum.

\section{B. Energy-Momentum Densities in Landau-Lifshitz Complex}

The non-zero components of $\ell^{a b c d}$ lead to the following components of energy and momentum (energy current) densities in Landau-Lifshitz complex 


$$
\begin{aligned}
L^{00} & =\frac{s}{2 \pi}\left[(2 s-1) \rho^{8 s^{2}}-\alpha^{2}\left(8 s^{3}-16 s^{2}+9 s-1\right)\right] \rho^{8 s^{2}-8 s-2}, \\
L^{i 0} & =0=L^{0 i} .
\end{aligned}
$$

This also yields momentum constant.

\section{Energy-Momentum Densities in Papapetrou Complex}

Here the components of energy and momentum (energy current) densities will become

$$
\begin{aligned}
& \Omega^{00}=\frac{s^{2} \rho^{8 s^{2}-2}}{2 \pi \alpha}\left[1-8 \alpha^{2}(s-1)^{2} \rho^{-8 s}\right], \\
& \Omega^{i 0}=0=\Omega^{0 i}
\end{aligned}
$$

which gives constant momentum.

\section{Energy-Momentum Densities in Möller Complex}

The energy-momentum densities turn out to be

$$
M_{a}^{b}=0
$$

giving a constant energy-momentum. We note that all the prescriptions provide constant momentum for this metric.

\section{SUMMARY AND DISCUSSION}

This paper continues the investigation of comparing various distributions presented in the literature. We have used four different prescriptions namely Einstein, Landau-Lifshitz, Papapetrou and Möller to calculate energy-momentum densities of two particular examples. These prescriptions turn out to be a powerful tool to evaluate energy-momentum for various physical systems. Although this work does not resolve the longstanding and crucial problem of the localization of energy in GR, but provides some information about it through such solutions. The following tables yield the non-zero components of the energy-momentum densities in each case. The notation EM has been used for Energy-Momentum.

Table 1(a). Weyl-Lewis-Papapetrou Metric: Einstein Complex

\begin{tabular}{|c|c|}
\hline EM Densities & Expressions \\
\hline$\Theta_{0}^{0}$ & $\frac{1}{8 \pi \rho}\left\{\gamma_{\rho}\left(e^{2 \gamma}-1\right)-\rho \gamma_{\rho \rho}+2 \psi_{\rho}+2 \rho \psi_{\rho \rho}-\rho \gamma_{z z}\right.$ \\
& $\left.+2 \rho \psi_{z z}+\frac{\omega_{\rho}^{2} e^{4 \psi}}{2 \rho}+\frac{\omega \omega_{\rho} e^{4 \psi}}{2 \rho}-\frac{\omega \omega_{\rho} e^{4 \psi}}{2 \rho^{2}}+\frac{2 \omega \omega_{\rho} \psi_{\rho} e^{4 \psi}}{\rho}\right\}$ \\
\hline & $\frac{y}{16 \pi \rho^{2}}\left[\left(\omega_{\rho \rho}+\omega_{z z}\right)+2 \omega\left(\gamma_{\rho \rho}+\gamma_{z z}\right)+2\left(\omega_{\rho} \gamma_{\rho}\right.\right.$ \\
& $\left.+\omega_{z} \gamma_{z}\right)-2 \omega\left\{2\left(\gamma_{\rho \rho}-2 \psi_{\rho \rho}\right)+\left(\gamma_{z z}-2 \psi_{z z}\right)\right\}$ \\
$\Theta_{1}^{0}$ & $-2 \omega\left\{\left(\gamma_{\rho}-2 \psi_{\rho}\right) \psi_{\rho}+\left(\gamma_{z}-2 \psi_{z}\right) \psi_{z}\right\}+\frac{4 \omega^{2} e^{4 \psi}}{\rho^{2}}\left(\omega_{\rho} \psi_{\rho}\right.$ \\
& $\left.+\omega_{z} \psi_{z}\right)+\frac{\omega^{2} e^{4 \psi}}{\rho^{2}}\left(\omega_{\rho \rho}+\omega_{z z}\right)+\frac{2 \omega e^{4 \psi}}{\rho}\left(\omega_{\rho}^{2}+\omega_{z}^{2}\right)$ \\
& $\left.-\frac{2 \omega_{\rho}}{\rho}+\frac{2 \omega}{\rho^{2}}+\frac{\omega_{\rho} e^{2 \gamma}}{\rho}+\frac{2 \omega \gamma_{\rho} e^{2 \gamma}}{\rho}-\frac{2 \omega e^{2 \gamma}}{\rho^{2}}-\frac{2 \omega^{2} \omega_{\rho} e^{4 \psi}}{\rho^{3}}\right]$ \\
\hline$\Theta_{2}^{0}$ & $-\frac{x}{16 \pi \rho^{2}}\left[\left(\omega_{\rho \rho}+\omega_{z z}\right)+2 \omega\left(\gamma_{\rho \rho}+\gamma_{z z}\right)+2\left(\omega_{\rho} \gamma_{\rho}\right.\right.$ \\
& $+2 \omega\left\{\left(\omega_{z} \gamma_{z}\right)-2 \omega\left\{2\left(\gamma_{\rho \rho}-2 \psi_{\rho \rho}\right)+\left(\gamma_{z z}-2 \psi_{z z}\right)\right.\right.$ \\
& $\left.+\omega_{z} \psi_{z}\right)+\frac{\omega^{2} e^{4 \psi}}{\rho^{2}}\left(\omega_{\rho \rho}+\omega_{z z}\right)+\frac{2 \omega e^{4 \psi}}{\rho}\left(\omega_{\rho}^{2}+\omega_{z}^{2}\right)$ \\
$\Theta_{0}^{2}$ & $\left.-\frac{2 \omega_{\rho}}{\rho}+\frac{2 \omega}{\rho^{2}}+\frac{\omega_{\rho} e^{2 \gamma}}{\rho}+\frac{2 \omega \gamma_{\rho} e^{2 \gamma}}{\rho}-\frac{2 \omega e^{2 \gamma}}{\rho^{2}}-\frac{2 \omega^{2} \omega_{\rho} e^{4 \psi}}{\rho^{3}}\right]$ \\
\hline$\Theta_{0}^{2}$ & $-\frac{y e^{4 \psi}}{16 \pi \rho^{2}}\left[\left(\omega_{\rho \rho}+\omega_{z z}\right)+4\left(\omega_{\rho} \psi_{\rho}+\omega_{z} \psi_{z}\right)\right]$ \\
\hline & $\frac{x e^{4 \psi}}{16 \pi \rho^{2}}\left[\left(\omega_{\rho \rho}+\omega_{z z}\right)+4\left(\omega_{\rho} \psi_{\rho}+\omega_{z} \psi_{z}\right)\right]$ \\
\hline
\end{tabular}


Table 1(b). Weyl-Lewis-Papapetrou Metric: Landau-Lifshitz Complex

\begin{tabular}{|c|c|}
\hline EM Densities & Expressions \\
\hline$L^{00}$ & $\begin{array}{c}\frac{1}{16 \pi \rho^{2}}\left[4 \rho\left(\gamma_{\rho}-\psi_{\rho}\right) e^{(\gamma-\psi)}-4 \rho\left(\gamma_{\rho}-2 \psi_{\rho}\right) e^{2(\gamma-2 \psi)}\right. \\
\quad-2\left\{\left(\gamma_{\rho \rho}-2 \psi_{\rho \rho}\right)+\left(\gamma_{z z}-2 \psi_{z z}\right)\right\} e^{2(\gamma-2 \psi)} \\
-4\left\{\left(\gamma_{\rho}-2 \psi_{\rho}\right)^{2}+\left(\gamma_{z}-2 \psi_{z}\right)^{2}\right\} e^{(\gamma-2 \psi)}+\frac{2 e^{2 \gamma}}{\rho^{2}}\left\{\omega\left(\omega_{\rho \rho}\right.\right. \\
\left.+\omega_{z z}\right)+2\left(\omega_{\rho}^{2}+\omega_{z}^{2}\right)+4 \omega\left(\omega_{\rho} \gamma_{\rho}+\omega_{z} \gamma_{z}\right)+\omega^{2}\left(\gamma_{\rho \rho}\right. \\
\left.\left.\left.\quad+\gamma_{z z}\right)+2 \omega^{2}\left(\gamma_{\rho}^{2}+\gamma_{z}^{2}\right)-\frac{2 \omega \omega_{\rho}}{\rho}-\frac{2 \omega^{2} \gamma_{\rho}}{\rho}+\frac{\omega^{2}}{\rho^{2}}\right\}\right]\end{array}$ \\
\hline$L^{10}=L^{01}$ & $\begin{array}{c}-\frac{y e^{2 \gamma}}{16 \pi \rho^{2}}\left[\left(\omega_{\rho \rho}+\omega_{z z}\right)+4\left(\omega_{\rho} \gamma_{\rho}+\omega_{z} \gamma_{z}\right)\right. \\
\left.+2 \omega\left(\gamma_{\rho \rho}+\gamma_{z z}\right)+4 \omega\left(\gamma_{\rho}^{2}+\gamma_{z}^{2}\right)-\frac{\omega_{\rho}}{\rho}-\frac{2 \omega \gamma_{\rho}}{\rho}\right]\end{array}$ \\
\hline$L^{20}=L^{02}$ & $\begin{array}{c}\frac{x e^{2 \gamma}}{16 \pi \rho^{2}}\left[\left(\omega_{\rho \rho}+\omega_{z z}\right)+4\left(\omega_{\rho} \gamma_{\rho}+\omega_{z} \gamma_{z}\right)\right. \\
\left.+2 \omega\left(\gamma_{\rho \rho}+\gamma_{z z}\right)+4 \omega\left(\gamma_{\rho}^{2}+\gamma_{z}^{2}\right)-\frac{\omega_{\rho}}{\rho}-\frac{2 \omega \gamma_{\rho}}{\rho}\right]\end{array}$ \\
\hline
\end{tabular}

Table 1(c). Weyl-Lewis-Papapetrou Metric: Papapetrou Complex

\begin{tabular}{|c|c|}
\hline EM Densities & Expressions \\
\hline$\Omega^{00}$ & $\begin{array}{c}\frac{e^{2 \gamma}}{8 \pi \rho}\left[\left(1-e^{-4 \psi}\right) \gamma_{\rho}+\left\{2 \psi_{\rho}-\rho\left(\gamma_{\rho \rho}-2 \psi_{\rho \rho}\right.\right.\right. \\
\left.\left.+\gamma_{z z}-2 \psi_{z z}\right)\right\} e^{-4 \psi}-2 \rho\left\{\left(\gamma_{\rho}-2 \psi_{\rho}\right)^{2}\right. \\
\left.+\left(\gamma_{z}-2 \psi_{z}\right)^{2}\right\} e^{-4 \psi}+\frac{1}{\rho}\left(\omega_{\rho}^{2}+\omega_{z}^{2}\right)+\frac{2 \omega^{2}}{\rho}\left(\gamma_{\rho}^{2}+\gamma_{z}^{2}\right) \\
+\frac{\omega}{\rho}\left(\omega_{\rho \rho}+\omega_{z z}\right)+\frac{\omega^{2}}{\rho}\left(\gamma_{\rho \rho}+\gamma_{z z}\right) \\
\left.+\frac{4 \omega \omega_{\rho}}{\rho}\left(\omega_{\rho} \gamma_{\rho}+\omega_{z} \gamma_{z}\right)+\frac{2 \omega^{2}}{\rho^{3}}-\frac{3 \omega^{2} \gamma_{\rho}}{\rho^{2}}-\frac{3 \omega \omega_{\rho}}{\rho^{2}}\right]\end{array}$ \\
\hline$\Omega^{10}=\Omega^{01}$ & $\begin{array}{c}-\frac{y e^{2 \gamma}}{16 \pi \rho^{2}}\left[\left(\omega_{\rho \rho}+\omega_{z z}\right)+4\left(\omega_{\rho} \gamma_{\rho}+\omega_{z} \gamma_{z}\right)\right. \\
\left.+2 \omega\left(\gamma_{\rho \rho}+\gamma_{z z}\right)+4 \omega\left(\gamma_{\rho}^{2}+\gamma_{z}^{2}\right)-\frac{\omega_{\rho}}{\rho}-\frac{2 \omega \gamma_{\rho}}{\rho}\right]\end{array}$ \\
\hline$\Omega^{20}=\Omega^{02}$ & $\begin{array}{c}\frac{x e^{2 \gamma}}{16 \pi \rho^{2}}\left[\left(\omega_{\rho \rho}+\omega_{z z}\right)+4\left(\omega_{\rho} \gamma_{\rho}+\omega_{z} \gamma_{z}\right)\right. \\
\left.+2 \omega\left(\gamma_{\rho \rho}+\gamma_{z z}\right)+4 \omega\left(\gamma_{\rho}^{2}+\gamma_{z}^{2}\right)-\frac{\omega_{\rho}}{\rho}-\frac{2 \omega \gamma_{\rho}}{\rho}\right]\end{array}$ \\
\hline
\end{tabular}

Table 1(d). Weyl-Lewis-Papapetrou Metric: Möller Complex

\begin{tabular}{|c|c|}
\hline EM Densities & Expressions \\
\hline$M_{0}^{0}$ & $\frac{1}{4 \pi}\left(\psi_{\rho}+\rho \psi_{\rho \rho}+\rho \psi_{z z}\right)+\frac{e^{4 \psi}}{8 \pi \rho}\left[\omega\left(\omega_{\rho \rho}+\omega_{z z}\right)\right.$ \\
& $\left.+4\left(\omega_{\rho} \psi_{\rho}+\omega_{z} \psi_{z}\right)+\left(\omega_{\rho}^{2}+\omega_{z}^{2}\right)-\frac{\omega \omega_{\rho}}{\rho}\right]$ \\
\hline & $-\frac{e^{4 \psi}}{8 \pi \rho}\left[\left(\omega^{2}+\rho^{2}\right)\left(\omega_{\rho \rho}+\omega_{z z}\right)+4\left(\omega^{2}+\rho^{2}\right)\left(\omega_{\rho} \psi_{\rho}\right.\right.$ \\
& $\left.+\omega_{z} \psi_{z}\right)+2 \omega\left(\omega_{\rho}^{2}+\omega_{z}^{2}\right)+4 \omega \rho^{2}\left(\psi_{\rho \rho}+\psi_{z z}\right)$ \\
$M_{2}^{0}$ & $\left.+4 \rho \omega \omega_{\rho}+\omega_{\rho}-\frac{\omega^{2} \omega_{\rho}}{\rho}\right]$ \\
\hline$M_{0}^{2}$ & $\frac{e^{4 \psi}}{8 \pi \rho}\left[\omega_{\rho \rho}+\omega_{z z}+4\left(\omega_{\rho} \psi_{\rho}+\omega_{z} \psi_{z}\right)-\frac{\omega_{\rho}}{\rho}\right]$ \\
\hline
\end{tabular}


Table 2. Levi-Civita Metric

\begin{tabular}{|c|c|}
\hline Prescription & Energy-Momentum Densities \\
\hline Einstein & $\Theta_{0}^{0}=\frac{s^{2} \rho^{8 s^{2}-2}}{2 \pi \alpha}, \Theta_{i}^{0}=0=\Theta_{0}^{i}$ \\
\hline \multirow{3}{*}{ Landau-Lifshitz } & $\begin{array}{c}L^{00}=\frac{1}{16 \pi}\left[\rho^{16 s^{2}-8 s-2}\left(16 s^{2}-8 s\right)\right. \\
\left.-2 \alpha^{2}\left(16 s^{2}-16 s-1\right) \rho^{8 s^{2}-8 s-2}\right] \\
\end{array} \quad L^{i 0}=0=L^{0 i}$ \\
\hline Papapetrou & $\begin{array}{c}\Omega^{00}=\frac{s^{2} \rho^{8 s^{2}-2}}{2 \pi \alpha}\left[1-8 \alpha^{2}(s-1)^{2} \rho^{-8 s}\right] \\
\Omega^{i 0}=0=\Omega^{0 i}\end{array}$ \\
\hline Möller & $M_{a}^{b}=0$ \\
\hline
\end{tabular}

From these tables, it is concluded that the energy-momentum densities turn out to be finite and well-defined in all the prescriptions for the spacetimes under consideration. In WeylLewis-Papapetrou metric, the non-vanishing momentum densities turn out to be the same in Landau-Lifshitz and Papapetrou complexes while the energy is different in each complex. It is worth mentioning here that for $\omega=0$ the results reduce to the case of the Weyl metric as found in the paper [31]. The energy for the Levi-Civita metric is different while momentum becomes constant in each prescription. It is worth mentioning that energy-momentum becomes constant for $s=0$ as expected for Minkowski spacetime.

We would like to remark that the results of energymomentum distribution for different spaceimes are not surprising. They support the fact that different energymomentum complexes, which are pseudo-tensors, are not covariant objects. This is in accordance with the equivalence principle [3] which implies that the gravitational field cannot be detected at a point. In GR, many energy-momentum expressions (reference frame dependent pseudo-tensors) have been proposed. There is no consensus as to which is the best. However, each expression has a geometrically and physically clear significance associated with the boundary conditions.
The difference of results supports the well-defined proposal developed by Cooperstock [36] and verified by many authors [28-31,37]. It is mentioned here that the results of the WeylLewis-Papapetrou metric found in teleparallel theory of gravity do not coincide with the results in GR [38].

Finally, we would like to mention that Virbhadra found [22] energy-momentum distribution of the Kerr-Newman metric by using Einstein, Landau-Lifshitz, Tolman and Möller energy-momentum complexes. He concluded that the four prescriptions could give the same result for the Kerr-Newman spacetime if appropriate coordinates are used. As is wellknown, Einstein, Landau-Lifshitz, Tolman's prescriptions can give meaningful results only if Cartesian coordinates are used but Möller's prescription does not require any such condition. We have followed the same coordinate system to obtain the energy-momentum for the two spacetimes. One can recover the results only if coordinates can be defined such that the metric can be made compatible. This is not always possible.

\section{Acknowledgment}

We would like to thank Mr. Jamil Amir for useful discussions on the subject during its write up.
[1] R. C. Tolman, Phys. Rev. 35, 8 (1930).

[2] A. Komar, Phys. Rev. 127, 1411 (1962); ibid 129, 1873 (1963); R. Penrose, Proc. R. Soc. London A 381, 53 (1982); In Asymptotic Behavior of Mass and Spacetime Geometry, ed. Flaherty, J.F. (Springer, Berlin, 1984).

[3] C. W. Misner, K. S. Thorne, and J. A. Wheeler, Gravitation (Freeman, New York, 1973).

[4] F. I. Cooperstock and R. S. Sarracino, J. Phys. A: Math. Gen. 11, 877 (1978)

[5] A. Trautman, Gravitation: An Introduction to Current Research, ed. Witten, L. (Wiley, New York, 1962).

[6] R. C. Tolman, Relativity, Thermodynamics and Cosmology (Oxford University Press, London, 1934).

[7] L. D. Landau and E. M. Lifshitz, The Classical Theory of Fields (Pergamon, Oxford, 1980).

[8] A. Papapetrou, Proc. R . Irish Acad. A 52, 11 (1948).

[9] P. G. Bergmann and R. Thompson, Phys. Rev. 89, 400 (1953).

[10] S. Weinberg, Gravitation and Cosmology (Wiley, New York,
1972).

[11] H. Bondi, Proc. R. Soc. London A 427, 249 (1990).

[12] R. Penrose, Proc. R. Soc. London A 388, 457 (1982); GR 10 Conference eds. Bertotti, B. de Felice, F. and Pascolini, A. Padova, 1, 607 (1983).

[13] J. D. Brown and J. W. York, Jr. Phys. Rev. D 47, 1407 (1993).

[14] S. A. Hayward, Phys. Rev. D 497, 831 (1994).

[15] G. Bergqvist, Class. Quantum Grav. 9, 1753 (1992).

[16] C. C. Chang, J. M. Nester, and C. Chen, Phys. Rev. Lett. 83, 1897 (1999).

[17] C. Möller, Ann. Phys. (NY) 4, 347 (1958).

[18] C. Möller, Ann. Phys. (NY) 12, 118 (1961).

[19] G. Lessner, Gen. Rel. Grav. 28, 527 (1996).

[20] D. Kovacs, Gen. Rel. Grav. 17, 927 (1985); J. Novotny, Gen. Rel. Grav. 19, 1043 (1987).

[21] A. Komar, Phys. Rev. 113, 934 (1959).

[22] K. S. Virbhadra, Phys. Rev. D 41, 1086 (1990); ibid 42, 1066 (1990); ibid 42, 2919 (1990). 
[23] J. M. Aguirregabiria, A. Chamorro, and K. S. Virbhadra, Gen. Rel. Grav. 28, 1393 (1996).

[24] S. S. Xulu, Int. J. of Mod. Phys. A 15, 1979 (2000); Mod. Phys. Lett. A 15, 1151 (2000).

[25] S. S. Xulu, Astrophys. Space Sci. 283, 23 (2003).

[26] A. Chamorro and K. S. Virbhardra, Int. J. of Mod. Phys. D 5, 251 (1994).

[27] S. S. Xulu, Int. J. of Mod. Phys. D 7, 773 (1998).

[28] M. Sharif, Int. J. of Mod. Phys. A 17, 1175 (2002).

[29] M. Sharif, Int. J. of Mod. Phys. A 18, 4361 (2003); ibid 19, 1495 (2004).

[30] M. Sharif and T. Fatima, Int. J. of Mod. Phys. A 20, 4309 (2005).

[31] M. Sharif and T. Fatima, Nouvo Cimento B 120, 533 (2005).

[32] R. D'Inverno, Introducing Einstein's Relativity (Oxford Univer- sity Press, 1995)

[33] H. Stephani, D. Kramer, M. A. H. MacCallum, C. Hoenselaers, and E. Hearlt, Exact Solutions of Einstein's Field Equations (Cambridge University Press, 2003).

[34] A. D. Konkowski, M. T. Helliwell, and C. Wieland, Gravitation and Cosmology: Proc. of the Spanish Relativity Meeting 2002, ed. A. Lobo (University of Barcelona Press, 2003)193.

[35] L. Herrera, M. F. Paiva, and O. N. Santos, J. Math. Phys. 40, 4064 (1999).

[36] F. I. Cooperstock, Annals Phys. 282, 215 (2000); Found. Phys. 22, 1011 (1992); 33, 1033 (2003).

[37] Thomas Bringly, Mod. Phys. Lett. A 17, 157 (2002); Found. Phys. 22, 1011 (1992); 33, 1033 (2003).

[38] M. Sharif and M. J. Amir, Mod. Phys. Lett. A 22, 425 (2007). 